\title{
Suicide Gene-Mediated Sequencing Ablation Revealed the Potential Therapeutic Mechanism of Induced Pluripotent Stem Cell-Derived Cardiovascular Cell Patch Post-Myocardial Infarction
}

\author{
Yuhua Wang, ${ }^{1, *}$ Wei Huang,, Jialiang Liang,, Zhili Wen,, Dehua Chang, Kai Kang,
} Jiapeng Wang, Meifeng Xu, Ronald W. Millard, ${ }^{2}$ and Yigang Wang ${ }^{1}$

\begin{abstract}
Aims: This study is designed to assess the protective cardiac effects after myocardial infarction (MI) of (i) cardiovascular progenitor cells (PC) differentiated directly into cardiomyocytes (CM) and endothelial cells (ECs) at the injury site, as separable from the effects of (ii) paracrine factors released from PC. Results: In vivo: bi-cell patch containing induced pluripotent stem cell (iPSC)-derived CM and EC (BIC) was transplanted onto the infarcted heart. BIC were transduced with herpes simplex virus thymidine kinase "suicide" gene driven by cardiac $N C X 1$ or endothelial vascular endothelium-cadherin promoter. IGF-1 $\alpha$ and VEGF levels released from ischemic tissues were significantly enhanced in the BIC patch treatment group. Heart function, infarction size, and vessel density were significantly improved after BIC patch treatment. These effects were completely abolished in the group given ganciclovir (GCV) at week 1 as a suicide gene activator, and partially abolished in the group given GCV at week 3 as compared with the untreated cell patch group. Innovation: This study was designed to distinguish between cell-based and noncell-based therapeutic effects of PC lineages after MI. PCs derived from iPSC were genetically modified to express "suicide" gene. iPSC-derived CM and EC were then ablated in situ at week 1 and 3 by intraperitoneal administration of GCV. This enabled direct assessment of the effects of iPSC transplantation on myocardial function and tissue regeneration potential. Conclusions: Data support a mechanism in which iPSC-derived cardiovascular lineages contribute directly to improved cardiac performance and attenuated remodeling. Paracrine factors provide additional support to the restoration of heart function. Antioxid. Redox Signal. 21, 2177-2191.
\end{abstract}

\section{Introduction}

$\mathbf{M}$ YOCARDIAL INFARCTION (MI) is a major therapeutic target for experimental progenitor cell (PC)-based therapies, as it results from interruption of blood supply to a region of the heart, causing heart cells to be damaged or die and evokes cardiac tissue remodeling. Recent studies have shown that induced pluripotent stem cells (iPSC) may have therapeutic potential (13). Experimental studies in animals have yielded encouraging results when iPSC are transplanted into peri-infarction myocardial zones (4). Treatments with iPSC appear to result in an improvement in cardiac contractile function (4), but numerous questions remain unanswered with regard to the use of such cells as therapy for post-MI repair. The mechanism by which post-MI cardiac function restoration occurs after iPSC treatment is currently unknown. It has been confirmed that PCs secrete cytokines, chemokines, and growth factors that could potentially promote repair of injured cardiac tissue (10). Two mechanisms have been suggested by which cardiac repair after an

Departments of ${ }^{1}$ Pathology and Laboratory Medicine and ${ }^{2}$ Pharmacology and Cell Biophysics, University of Cincinnati Medical Center, Cincinnati, Ohio.

*These authors contributed equally to this work. 


\section{Innovation}

Our experimental data provide new insights into the role of cell-based versus noncell-based therapeutic effects of progenitor cells (PC) derived from induced pluripotent stem cells (iPSC). Current research inadequately distinguishes the nature of post-MI restoration of cardiac function with cell-based therapies. Our focus on noncellbased therapy mediated by paracrine factors secreted by PCs is supported by several studies in which PCs that secrete cytokines, chemokines, and growth factors are observed to improve heart function. However, increasing evidence supports the notion that iPSC differentiation into cardiovascular cell lineages is important to compensate for pathological insufficiency and to prolong the therapeutic effect, leading to a favorable reversal of tissue remodeling after ischemic conditions.

ischemic insult can occur: (i) PC differentiation directed toward a coordinated generation of new cardiac muscle cells and accompanying establishment of a new or expanded microcirculation (7) and (ii) PC secretions of paracrine factors, including cytokines, chemokines, and growth factors that alone or in combination recruit new reparative cells to the injury area and promote and support in situ tissue repair process $(4,7,10,13)$. The latter paracrine mechanism could potentially provide for a noncell-based alternative to the PC use in treatment of cardiovascular disease (18). Certainly, delivery of a paracrine agent might be preferable to cellbased therapies, as such molecular entities are generally easier to produce and could be safer as they cannot replicate or differentiate. However, since iPSC can be programmed to differentiate directly into specific and desired cardiovascular cell lineages, these cell-based approaches have recently gained interest as potential therapeutic treatments $(4,12)$.

The present study seeks to determine whether iPSCproduced therapeutic effects in postischemic myocardium can be ascribed preferentially to a cell-based differentiation or to a cell-derived product mechanism. To obtain evidence on the respective roles of these two mechanisms, an "inducible suicide gene" approach was employed. iPSC-derived cardiovascular PCs were genetically modified to express thymidine kinase (TK) "suicide" gene driven by cardiac promoter (NCX1) and vascular endothelium ([VE]-cadherin) promoter in cardiac myocytes and endothelial cell (EC) lineages, respectively. When these cells are exposed to GCV (8), the pro-drug GCV will be converted into a triphosporylated cytotoxic agent by TK, thereby triggering the death of such cells. Thus, by specifically targeting iPSCderived $\mathrm{CM}$ and $\mathrm{EC}$ at different time windows with a sequential administration of GCV, the study design enables an assessment of the role of these two mechanisms mentioned earlier in any observed cardiac tissue repair process.

\section{Results}

\section{Transduction of TK for suicide gene delivery}

The lentiviral vectors co-expressing green fluorescent protein (GFP) and TK under the control of promoterless (pCDH-Null-TK), CVM promoter (pCDH-CMV-TK), and specific cardiac sodium-calcium exchanger (NCX1) pro- moter (pCDH-NCX1-TK) were constructed, as shown in Figure 1A-C, respectively. Neonatal cardiomyocytes (Neo$\mathrm{CM}$ ) were transfected with lentivirus containing the herpes simplex virus TK under the control of $N C X 1$ promoter, or CMV promoter, or promoterless vector (Null) as control, respectively. TK expressions in Neo-CM were assessed by reverse transcription-polymerase chain reaction (RT-PCR) (Fig. 1E). TK was expressed specifically in Neo-CM ${ }^{\text {CMV-TK }}$ and $\mathrm{Neo}-\mathrm{CM}^{\mathrm{NCX} 1-\mathrm{TK}}$ but not in the Neo-CM ${ }^{\text {Null-TK }}$ group (Fig. 1E). CM derived from iPSC (CM) were transduced with TK gene and then treated with vehicle or ganciclovir (GCV, $100 \mu M)$. Two days later, cells were photographed under a fluorescent microscope (Fig. 1G). CM ${ }^{\text {Null-TK}}, \mathrm{CM}^{\mathrm{CMV}-\mathrm{TK}}$, and $\mathrm{CM}^{\mathrm{NCX} 1-\mathrm{TK}}$ survived when $\mathrm{CM}$ was treated with a vehicle. However, all $\mathrm{CM}^{\mathrm{CMV}-\mathrm{TK}}$ and $\mathrm{CM}^{\mathrm{NCX} 1-\mathrm{TK}}$ died when GCV $(100 \mu M)$ was administered.

Similarly, the lentiviral vectors co-expressing GFP and TK under the control of promoterless (pCDH-Null-TK), CVM promoter (pCDH-CMV-TK), and specific EC promoter (VEcadherin) were constructed as shown in (Fig. 1A, B, D, respectively). TK expression in ECs was detected by RT-PCR (Fig. 1F). TK gene was expressed in ECs ${ }^{\text {VE-TK }}$ and ECs ${ }^{\mathrm{CMV}-}$ ${ }^{\text {TK }}$ but not in ECs ${ }^{\text {Null-TK }}$ (Fig. 1F). ECs derived from iPSC (EC) were then transduced with the lentivirus mentioned earlier. The cell growth of EC ${ }^{\text {Null-TK }}, \mathrm{EC}^{\mathrm{CMV}-\mathrm{TK}}$, or EC ${ }^{\mathrm{VE}-\mathrm{TK}}$ was not influenced when treated with vehicle. There were no surviving cells that expressed TK in the EC ${ }^{\mathrm{CMV}-\mathrm{TK}}$ or EC ${ }^{\mathrm{VE}-}$ TK group. In fact, the only group in which cells survived after

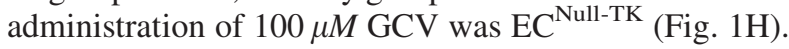

\section{Characteristics of iPSC-derived cardiovascular PCs}

The gene expressions of Oct4, Sox2, Klf4, GAGT4, $N k x 2.5$, and GAGT2 were assessed by quantitative RT-PCR (qRT-PCR) to investigate the phenotype of cardiovascular PCs derived from iPSC. The gene expression levels of Oct4, Sox2, and Klf4 were gradually decreased; while the GATA4, GATA2, and $N k \times 2.5$ were upregulated in a time-dependent manner (Fig. 2A). At 2 weeks after the formation of EBs, the expression level of the stem cell marker Oct4 decreased (Fig. $2 \mathrm{~B}$ ); whereas the percentages of $\alpha$-sarcomeric actin-positive cells and $\mathrm{CD}_{3}{ }^{+}$cells increased to $66.4 \%$ and $15.4 \%$, respectively, suggesting that $\mathrm{CM}$ and $\mathrm{EC}$ were successfully differentiated from iPSC. CM derived from iPSC were also confirmed by positive staining with the $\alpha$-sarcomeric actin antibody, a specific cardiomyocyte marker (Fig. 2C).

\section{Growth factor release in vitro and in vivo}

The gene expression of $I G F-1 \alpha$ and $V E G F$ was significantly upregulated, while $T G F-\beta 1$ expression was significantly reduced in $\mathrm{CM}$ after $4 \mathrm{~h}$ of exposure to anoxia as compared with levels detected in CM cultured in normoxia, $p<0.05$, (Fig. 3A). Similarly, all gene expression was significantly upregulated except $I G F-1 \alpha$, which was significantly reduced in EC after $4 \mathrm{~h}$ of anoxia as compared with EC cultured under normoxic conditions, $p<0.05$, (Fig. 3B).

The expression of $I G F-1 \alpha, V E G F$, and $T G F-\beta 1$ was assessed by Western blotting (Fig. 3C) to explore the growth factor-releasing profiles of infarcted hearts with various treatments. All growth factors were significantly upregulated in a time-dependent manner in the $\mathrm{MI}^{\mathrm{BIC}}$ (MI operated rats with bi-cell $(\mathrm{CM}+\mathrm{EC})$-seeded peritoneum patch) group as 
A

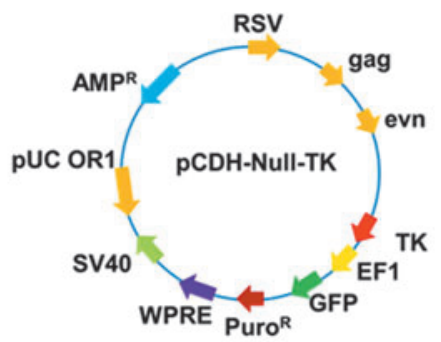

C

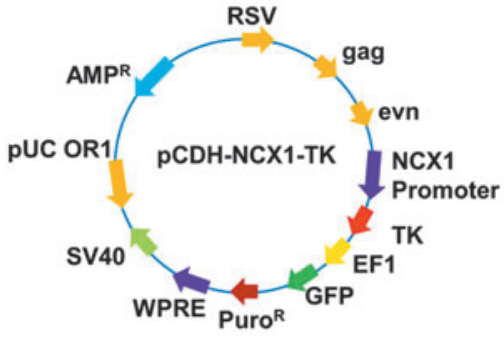

E

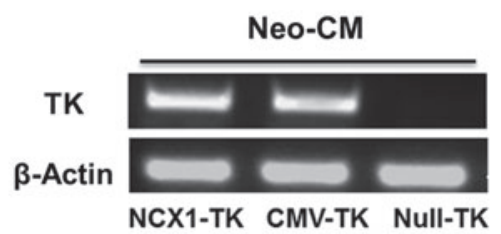

G

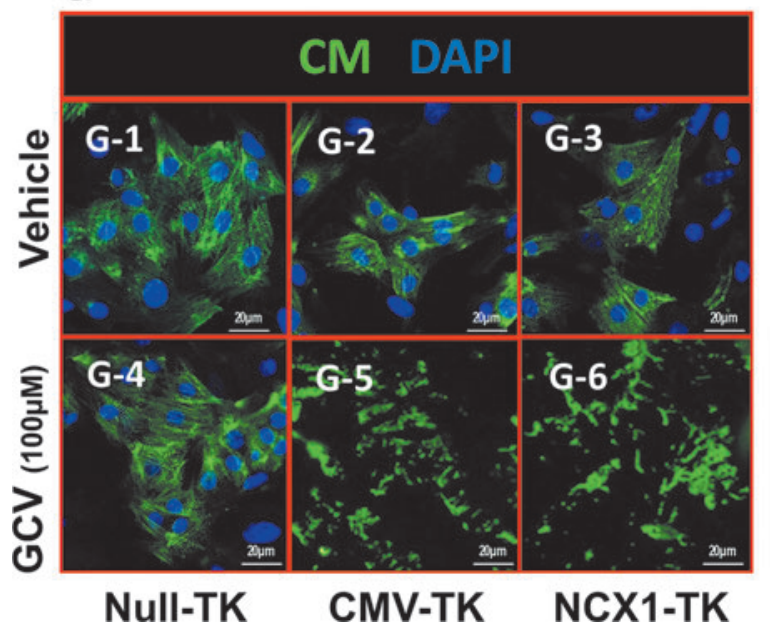

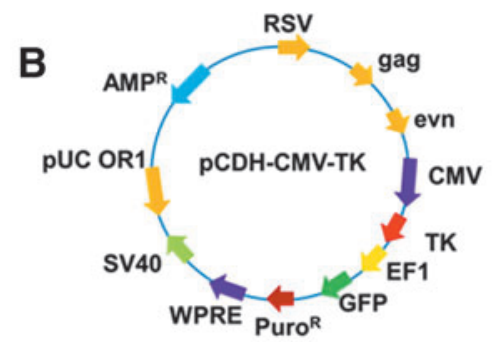

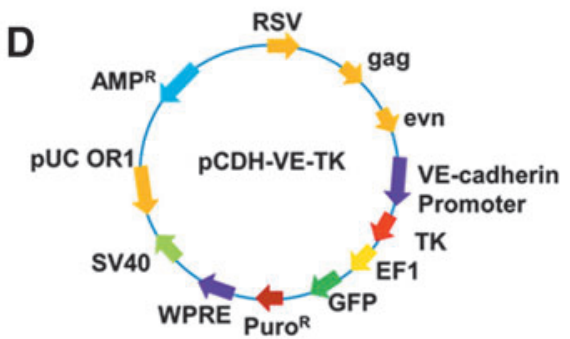

$\mathbf{F}$

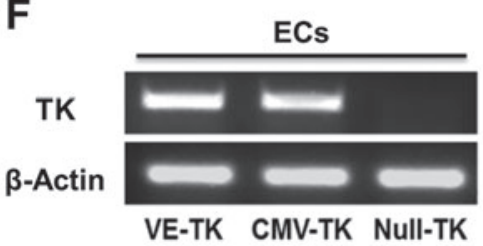

H

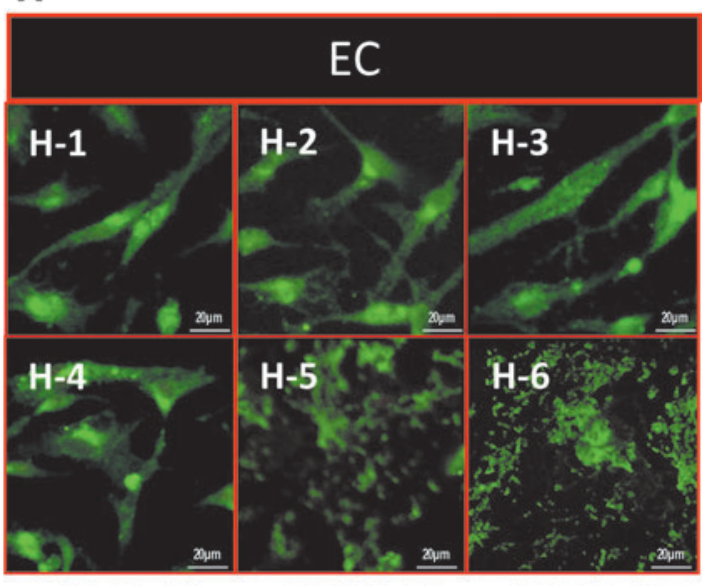

FIG. 1. Generation of suicide gene and its constructions. (A-D) The lentiviral vectors co-expressing green fluorescent protein (GFP) and thymidine kinase (TK) under the control of promoterless (pCDH-Null-TK), CVM promoter (pCDHCMV-TK), specific cardiac sodium-calcium exchanger (NCX1) promoter (pCDH-NCX1-TK), and specific endothelial cell (VE-cadherin) promoter (pCDH-NCX1-TK) were constructed, respectively. (E, F) Suicide gene (TK) expression in neonatal cardiomyocytes (Neo-CM) and endothelial cells (ECs) under the control of various promoters assessed by reverse transcription-polymerase chain reaction (RT-PCR). (G) The effect of suicide gene (TK) on induced pluripotent stem cell (iPSC)-derived CM (CM) under various promoters. CM were killed by ganciclovir (GCV) under the control of CVM promoter (CMV-TK) and NCX1 promoter (NCX1-TK), but were not killed by GCV under the control of promoterless (Null-TK). (H) The effect of suicide gene (TK) on iPSC-derived EC (EC) under various promoters. EC were killed by GCV under the control of CVM promoter (CMV-TK) or specific endothelial cell (VE-cadherin) promoter (VE-TK), but were not killed by GCV under the control of promoterless (Null-TK).

compared with the $\mathrm{MI}^{\mathrm{P}}$ group (MI operated rats with peritoneum patch without cells) (Fig. 3D-F). In addition, upregulation of growth factor(s) expression occurred immediately after BIC implantation and reached a peak level on day 7 (except for $T G F-\beta 1$, which peaked at day 3 ). Growth factor(s) gradually returned to similar levels in both $\mathrm{MI}^{\mathrm{BIC}}$ and $\mathrm{MI}^{\mathrm{P}}$ groups by day 28 (Fig. 3C).

The role of selective timed elimination of cardiovascular cells in growth factor release was also investigated. Before and 21 days after cell patch implantation, there was no 
A

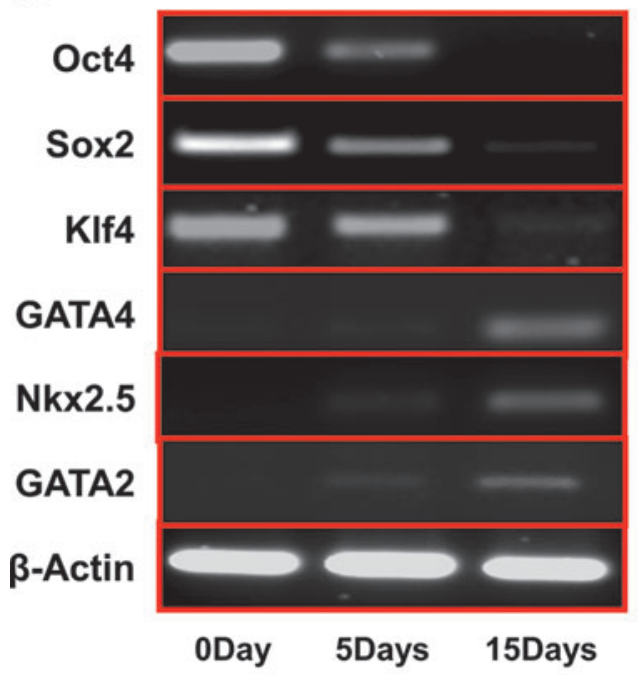

C

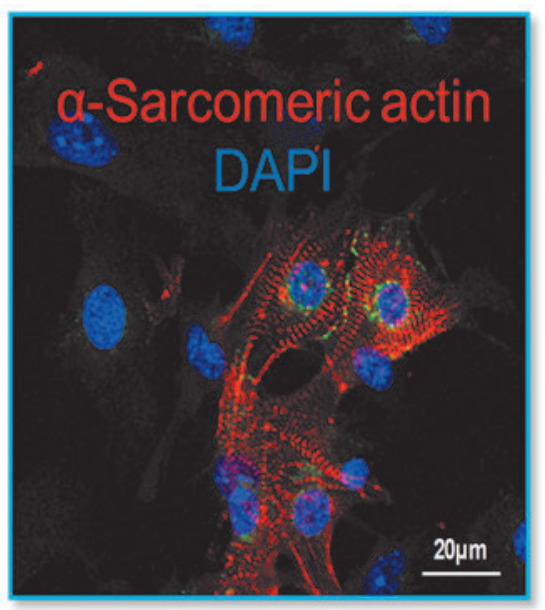

B
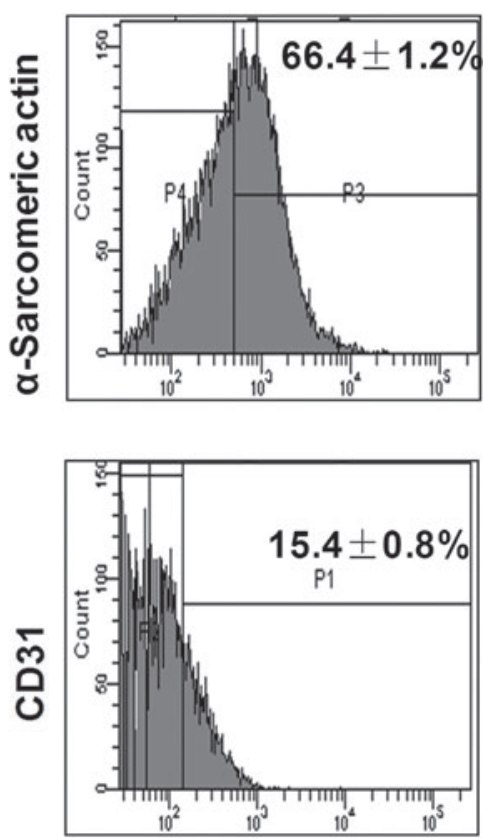

FIG. 2. Characteristics of iPSCderived cardiovascular and progenitor cells. (A) The gene expressions for Oct4, Sox2, Klf4 GAGT4, GAGT2, and Nkx2.5 were assessed by qPCR. (B) The expression of $\alpha$-sarcomeric actin, CD31, and Oct4-positive cells was assessed by flow cytometry. (C) CM derived from iPSC was also confirmed by staining of $\alpha$-sarcomeric actin antibody. significant difference in expression of $I G F-1 \alpha$ (Fig. 3G), $V E G F$ (Fig. 3H), and TGF- $\beta 1$ (Fig. 3I) in the various treatment groups. The expression of $I G F-1 \alpha, V E G F$, and $T G F-\beta 1$ was significantly reduced in the $\mathrm{MI}^{\mathrm{BIC}}+\mathrm{GCV} 1$ group (MIoperated rats with bi-cell patch given GCV in first week) in the first week. However, the increased levels of these growth factors $(I G F-1 \alpha, V E G F$, and $T G F-\beta 1)$ were not diminished at 7 days in the $\mathrm{MI}^{\mathrm{BIC}}+\mathrm{GCV} 3$ group (MI-operated rats with bicell patch given GCV in third week). These expression levels were similar to the $\mathrm{MI}^{\mathrm{BIC}}$ group, indicating that growth factors were mainly released during the first week.

\section{Evidence of angiomyogenesis}

Four weeks after cell patch treatment, capillary density, as determined by von Willebrand factor (vWF) staining, was significantly higher in the $\mathrm{MI}^{\mathrm{BIC}}+\mathrm{GCV} 3$ group than in the $\mathrm{MI}^{\mathrm{BIC}}+\mathrm{GCV} 1$ group at 4 weeks after BIC sheet implantation in situ. This result was similar to that observed in the $\mathrm{MI}^{\mathrm{P}}$ control group. Notably, capillary density was the highest in the $\mathrm{MI}^{\mathrm{BIC}}$ group as compared with any other group (Fig. 4A, C). In addition, GCV administration significantly attenuated the new vessel formation, leading to significantly decreased $\mathrm{GFP}^{+}$vessel number in the $\mathrm{MI}^{\mathrm{BIC}}+\mathrm{GCV} 1$ group as compared with the $\mathrm{MI}^{\mathrm{BIC}}+\mathrm{GCV} 3$ group. However, more $\mathrm{GFP}^{+}$ new vessels derived from iPSC were observed in the $\mathrm{MI}^{\mathrm{BIC}}$ group when compared with any other group (Fig. 4B, D).

Four weeks after cell patch placement, Masson's Trichrome staining confirmed that the patch was tightly adhered to the epicardial surface of the recipient heart. Specifically, as seen in low to high magnification images of the $\mathrm{MI}^{\mathrm{BIC}}$ group (Fig. 5A-I), large numbers of the CM were proliferating within the cell patch. Similarly, large numbers of the GFP ${ }^{+}$ cells were observed in the cell patch area in the $\mathrm{MI}^{\mathrm{BIC}}$ group (Fig. 5A-E), and were positively stained for $\alpha$-sarcomeric actin, a specific marker for $\mathrm{CM}$ (Fig. 5B, red colors), indicating that most of these $\mathrm{GFP}^{+}$cells were CM. The distinctive myofibrillar Z-line was also seen in these $\mathrm{GFP}^{+}$cells when viewed at high magnification (Fig. 5A). However, these new $\mathrm{GFP}^{+} \mathrm{CM}$ derived from iPSC were severely eliminated by GCV treatment in the third week, and no cardiomyocyte distinctive structures were observed in the transplanted cells in the $\mathrm{MI}^{\mathrm{BIC}} \mathrm{GCV} 3$ group (Fig. 5F). Similarly, CM characteristic $\mathrm{GFP}^{+}$cells were killed in the $\mathrm{MI}^{\mathrm{BIC}}$ GCV1 group 
FIG. 3. Growth factor release from $\mathrm{CM}$ and endothelial cell (EC) in vitro and in vivo. $(\mathrm{A}, \mathrm{B})$ Growth factor release in vitro. (A) The gene expression of $I G F-1 \alpha$, $V E G F$, and $T G F-\beta 1$ in CM. All values expressed as mean \pm SEM. $n=6$ for each group. (B) The gene expression of $I G F-1 \alpha, V E G F$, and $T G F-\beta 1$ in EC. ${ }^{*} p \leq 0.05$. All values expressed as mean \pm SEM. $n=6$ for each group. (C) The growth factor release in left ventricular tissue was assessed by Western blotting. (D) Quantitative data for $I G F-1 \propto$ expression at various time points with patch only (no cells) or with bi-cell (CM and EC) patch implantation. $* p \leq 0.05$. All values expressed as mean \pm SEM. $n=6$ for each group. (E) Quantitative data for VEGF expression at various time points. $* p \leq 0.05$. All values expressed as mean \pm SEM. $n=6$ for each group. (F) Quantitative data for $T G F-\beta 1$ expression at various time points. $* p \leq 0.05$ was considered statistically significant. All values expressed as mean \pm SEM. $n=6$ for each group. (G-I) The role of time in sequential selective elimination of cardiovascular cells in growth factor release. The expression of $I G F-1 \alpha, V E G F$, and $T G F-\beta 1$ in left ventricular tissue was analyzed at three different time points. $* p \leq 0.05$ was considered statistically significant. All values expressed as mean \pm SEM. $n=6$ for each group. $\mathrm{MI}^{\mathrm{P}}$ group: $\mathrm{MI}$-operated rats with peritoneum patch without cells; $\mathrm{MI}^{\mathrm{BIC}}$ group: MI-operated rats with bi-cell (CM +EC)-seeded peritoneum patch; $\mathrm{MI}^{\mathrm{BIC}}+\mathrm{GCV} 1$ group: MI-operated rats with bi-cell patch given GCV in first week; $\mathrm{MI}^{\mathrm{BIC}}+$ GCV1 group: MI-operated rats with bi-cell patch given GCV in third week.
A
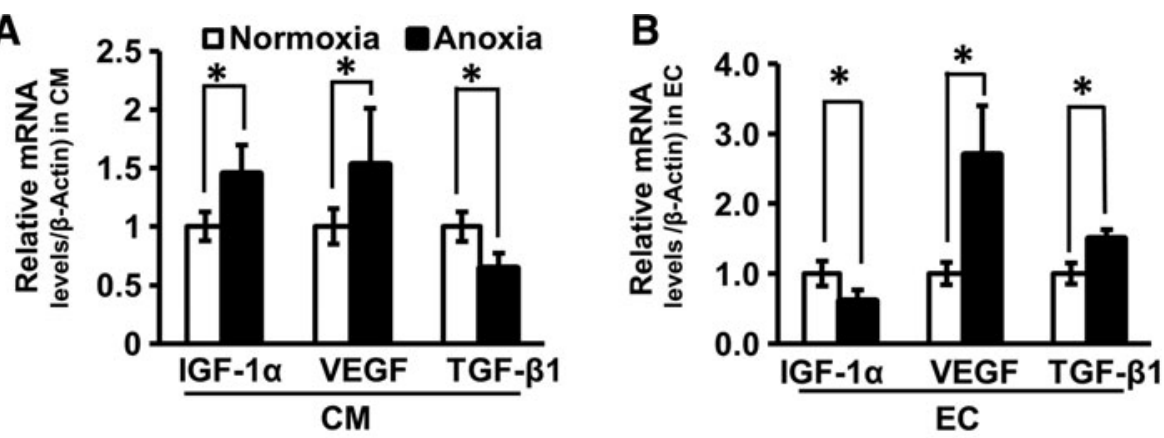

C
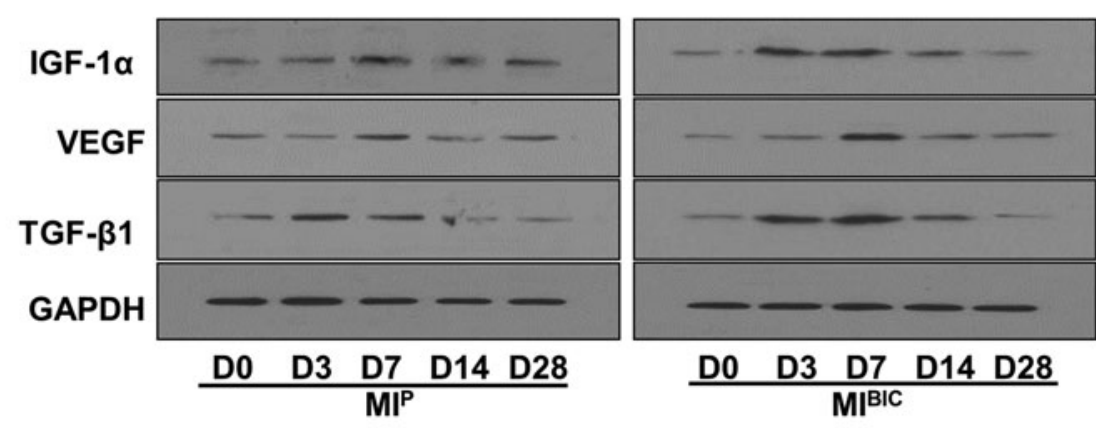

D

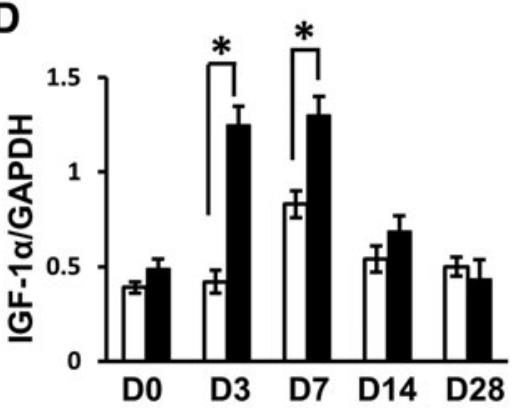

E

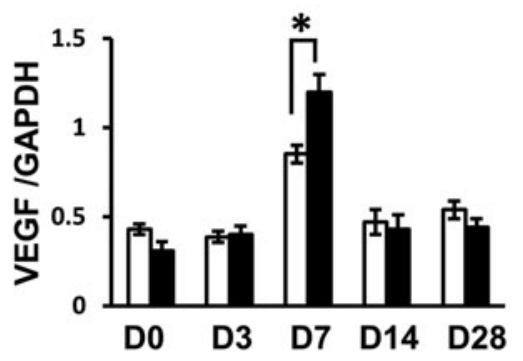

$\mathbf{F}$

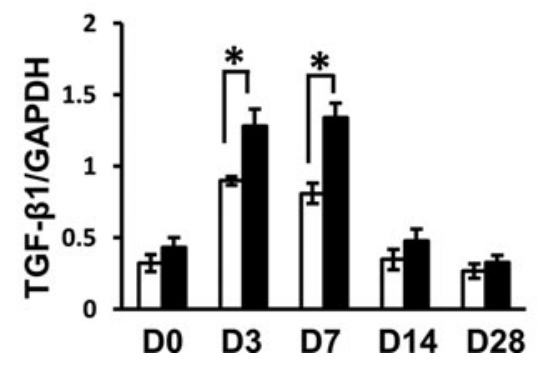

(data not shown). Quantitative analysis showed that the number of $\mathrm{GFP}^{+}$cells within the cell patch and peri-infarcted area of the heart were present to a greater extent in the $\mathrm{MI}^{\mathrm{BIC}}$ group in the absence of GCV administration than in any of the other groups, * $*_{p} \leq 0.05$, (Fig. $5 \mathrm{~J}, \mathrm{~K}$ ). The number of $\mathrm{GFP}^{+}$ cells was significantly reduced in $\mathrm{MI}^{\mathrm{BIC}} \mathrm{GCV} 1$ and $\mathrm{MI}^{\mathrm{BIC}}$ GCV3 groups after GCV treatment at the first or third week, suggesting that implanted cells were effectively killed by GCV treatment. Notably, histological assessment for an immune reaction against iPSC revealed no evidence of infiltration of $\mathrm{GFP}^{+}$iPSC-derived $C D 45^{+}$lymphocytes into the infarcted region (Fig. 5L).
Assessment of myocardial fibrosis area, myocyte hypertrophy, heart functional changes, and apoptosis after time-dependent elimination of implanted cells

Four weeks after cell patch implantation, the extent of MI was assessed using Masson's Trichrome staining in the various patch treatment groups (Fig. 6A, B). The percentage of left ventricular (LV) infarct area was significantly reduced in the $\mathrm{MI}^{\mathrm{BIC}}+\mathrm{GCV} 3$ group and was reduced further in the $\mathrm{MI}^{\mathrm{BIC}}$ group, while no remarkable changes were observed in infarct size/fibrosis area (as\% of LV) in the $\mathrm{MI}^{\mathrm{BIC}}+\mathrm{GCV} 1$ group as compared with the $\mathrm{MI}^{\mathrm{P}}$ group (Fig. 6B). 

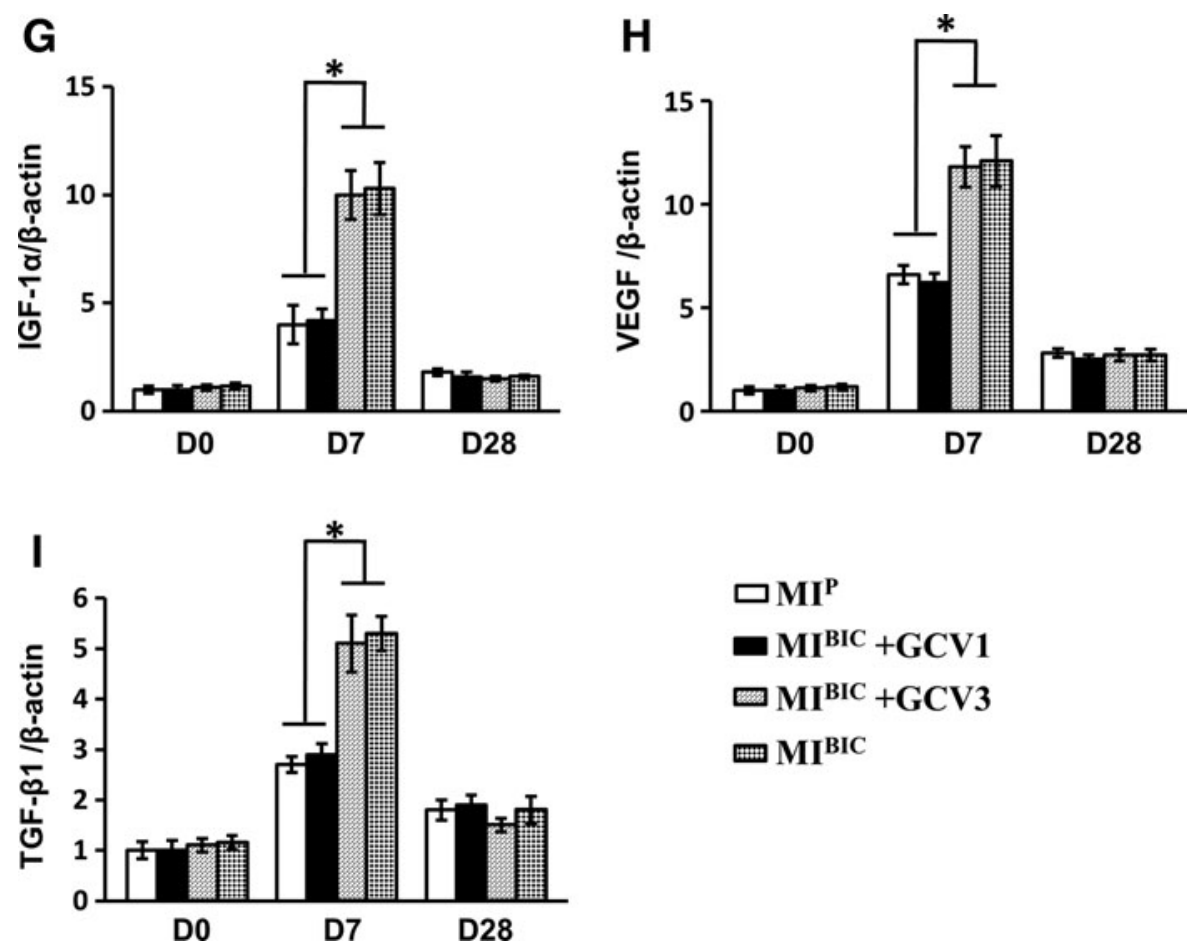

FIG. 3. (Continued).

A

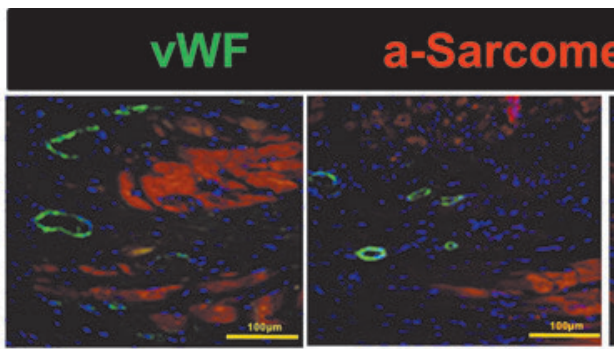

MIP

$M I^{\mathrm{BIC}}+\mathrm{GCV} 1$

B

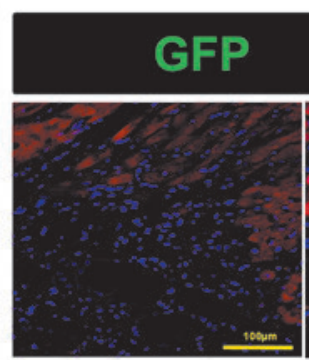

MIP

C

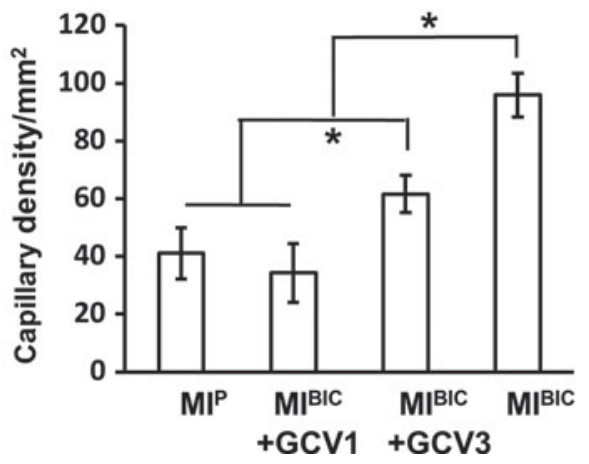

a-Sarcomeric actin

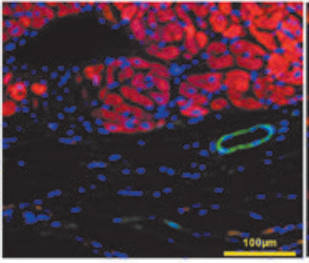

MIBIC+GCV1

$\mathrm{MI}^{\mathrm{BIC}}+\mathrm{GCV} 3$

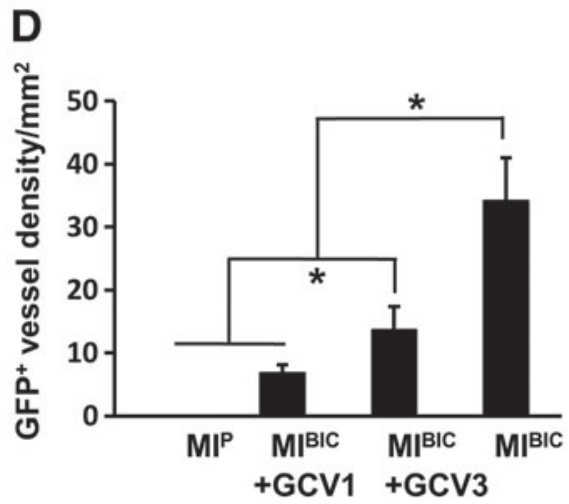

FIG. 4. Effect of selective, timed elimination of cardiovascular cells on angiogenesis. (A) Representative immunofluorescence staining for von Willebrand factor $(v W F)$ from rat hearts at 4 weeks after cell patch implantation was used to identify vessel density. DAPI was used to identify nuclei. $\alpha$ Sarcomeric actin was used to identify cardiomyocytes. Color codes given at the top. Scale bar $=100 \mu \mathrm{m}$. (B) Representative immunofluorescent staining for GFP-positive vessels from rat hearts at 4 weeks after cell path implantation. DAPI was used to identify nuclei. $\alpha$-Sarcomeric actin was used to identify cardiomyocytes. Color codes given at the top. Scale bar= $100 \mu \mathrm{m}$. (C, D) Quantitative data for capillary density (C) and new vessel density (D) responses in the four treatment groups reflect paracrine and cellular (CM and EC) contributions. All values were expressed as mean $\pm \mathrm{SEM}$. ${ }^{*} p<0.05$ was considered statistically significant; $n=6$ in each group. 
To determine cardiomyocyte hypertrophy at 4 weeks postMI, hearts were stained with $\alpha$-sarcomeric actin (for cardiomyocyte) and laminin (for surface cell membrane) (Fig. 6C). Of note, CM were the largest (Fig. 6D) and fewest in number (Fig. 6E) in the $\mathrm{MI}^{\mathrm{P}}$ group in comparison to other groups, indicating myocyte hypertrophy. However, with BIC treatment, cardiomyocyte hypertrophy was prominently attenuated, as evidenced by higher cardiomyocyte density and a decrease in cell surface area in the $\mathrm{MI}^{\mathrm{BIC}}$ group. This presumptive salutary effect of BIC was completely reversed by GCV administration at week $1\left(\mathrm{MI}^{\mathrm{BIC}}+\mathrm{GCV} 1\right)$, but only attenuated in the group given GCV at week $3\left(\mathrm{MI}^{\mathrm{BIC}}+\mathrm{GCV} 3\right)$ as compared with the $\mathrm{MI}^{\mathrm{BIC}}$ group.

Then, functional assessments (LVDd, LVDs, EF, and FS) are illustrated in Figure $6 \mathrm{H}-\mathrm{K}$ for the various treatment groups at 4 weeks after cell patch implantation. There were no significant differences in heart rate among the groups (data not shown) and no significant differences in LV function in terms of LVDd, LVDs, EF, and FS between the $\mathrm{MI}^{\mathrm{BIC}}+$ GCV1 group and the $\mathrm{MI}^{\mathrm{P}}$ control group. However, LVDd, LVDs, EF, and FS were significantly improved in the MI${ }^{\mathrm{BIC}}+\mathrm{GCV} 3$ group as compared with the MI ${ }^{\mathrm{P}}$ group. The most significant changes were observed in the $\mathrm{MI}^{\mathrm{BIC}}$ group, in which both LVDd and LVDs were significantly decreased, and an accompanying increase of EF and FS compared with any other groups. Although ventricular function was improved in the $\mathrm{MI}^{\mathrm{EC}}$ group and $\mathrm{MI}^{\mathrm{CM}}$ groups as compared with the $\mathrm{MI}^{\mathrm{P}}$ group, no significant differences were detected between the MI ${ }^{\mathrm{EC}}$ and $\mathrm{MI}^{\mathrm{CM}}$ groups in LVDd, LVDs, EF, and FS (Supplementary Fig. S1A-D; Supplementary Data are



FIG. 5. Characteristics of cardiomyocytes derived from iPSC in cell patch. (A-E) Fluorescence microscopy demonstrated that newly formed cardiomyocytes (green color) from infarcted areas at various magnifications in cardiovascular cell patch group at 4 weeks after cell graft in $\mathrm{MI}^{\mathrm{BIC}}$ group (data were not shown in other groups). All cardiomyocytes derived from iPSC show GFP-positive expression. In (B), $\alpha$-sarcomeric actin (red color) was used to further identify cardiomyocytes, and DAPI was used to identify all nuclei (blue color). EPI indicates the epicardial interface with the cell patch. (F-I) Cardiomyocytes derived from iPSC in the cell patch were completely destroyed by GCV treatment. Data shown here are at 4 weeks after cell patch in the $\mathrm{MI}^{\mathrm{BIC}}+\mathrm{GCV} 3$ group. Similar results were also observed in the MI ${ }^{\mathrm{BIC}}+\mathrm{GCV} 1$ group (data not shown). ( $\mathbf{J}-\mathbf{K})$ shows quantitative numbers of GFP-positive $\left(\mathrm{GFP}^{+}\right)$cells either in cell patches or in infarcted areas at 4 weeks after cell patch transplantation. All values were expressed as mean \pm SEM. $* p<0.05$ was considered statistically significant; $n=6$ in each group. (L) Representative immunofluorescence staining for CD45 from rat hearts at 4 weeks after cell patch implantation was used to identify vessel density. DAPI was used to identify nuclei. CD45 was used to identify inflammatory cells. Color codes given at the top. Scale bar $=100 \mu \mathrm{m}$.

$\mathrm{MI}^{\mathrm{P}}$ group: MI-operated rats with peritoneum patch without cells; $\mathrm{MI}^{\mathrm{BIC}}$ group: MI-operated rats with bi-cell $(\mathrm{CM}+\mathrm{EC})-$ seeded peritoneum patch; $\mathrm{MI}^{\mathrm{BIC}}+\mathrm{GCV} 1$ group: MI-operated rats with bi-cell patch given GCV in first week; $\mathrm{MI}^{\mathrm{BIC}}+$ GCV1 group: MI-operated rats with bi-cell patch given GCV in third week. 

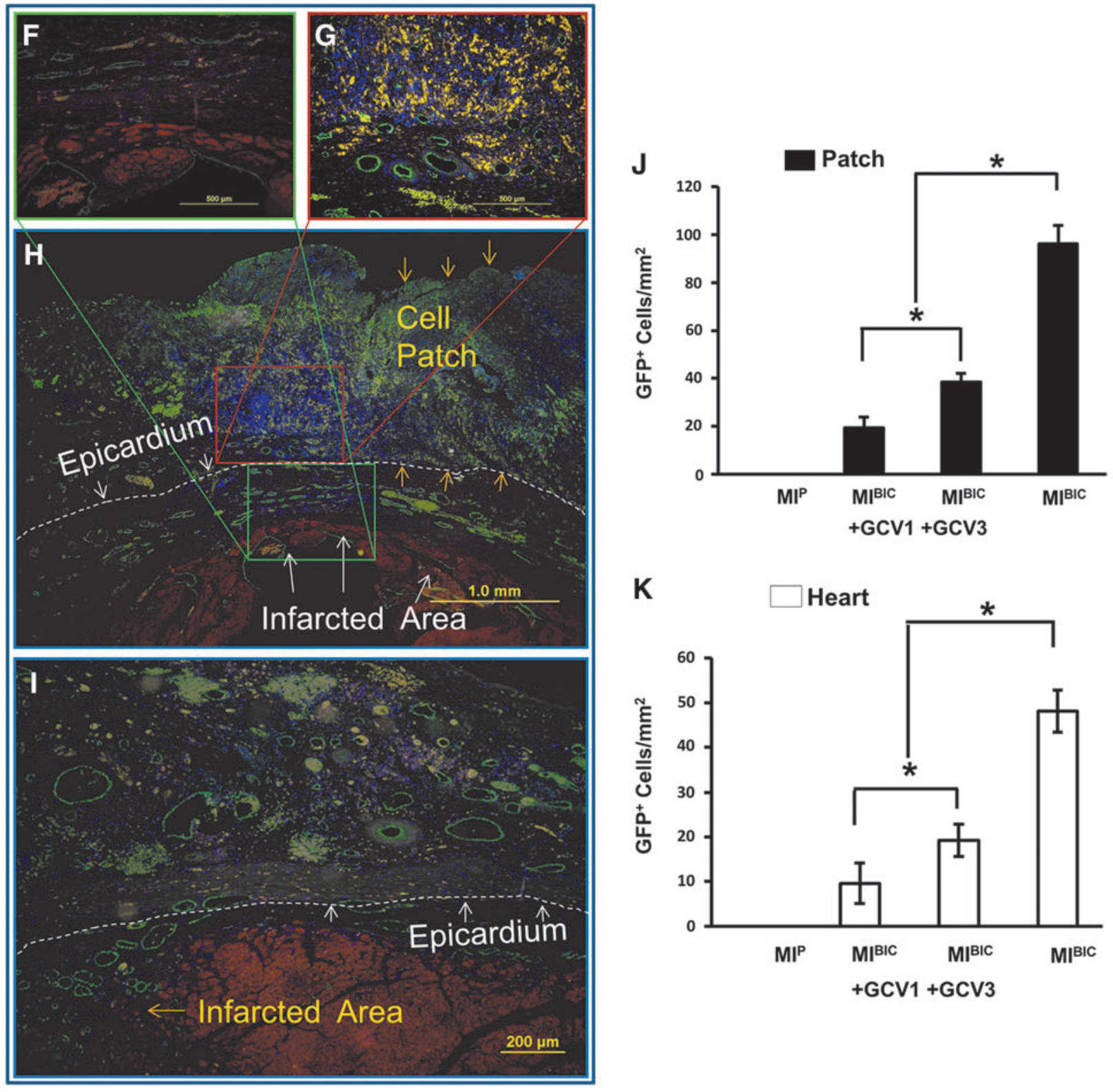

$\mathbf{L}$

\section{CD45}

\section{GFP}

DAPI

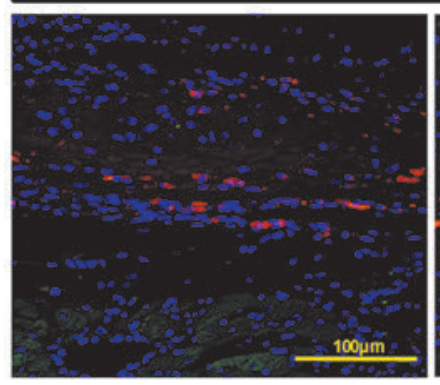

MIP

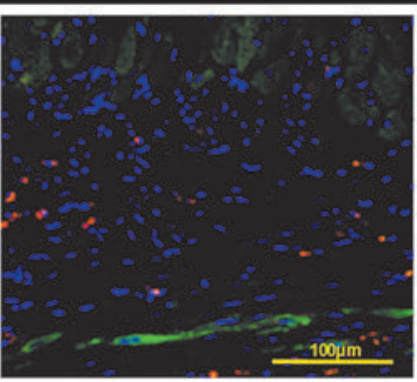

$\mathrm{MI}^{\mathrm{BIC}}+\mathrm{GCV} 1$

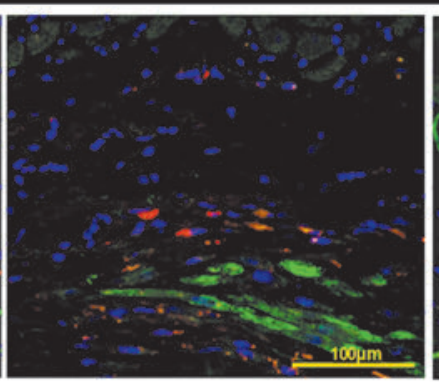

$\mathrm{MI}^{\mathrm{BIC}}+\mathrm{GCV} 3$

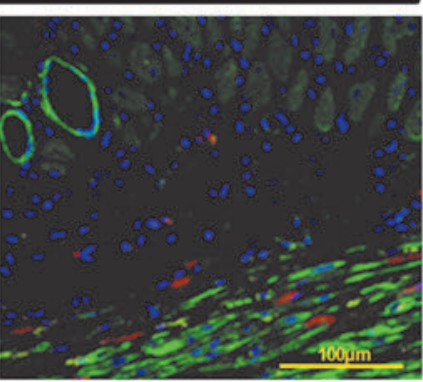

MIBIC

FIG. 5. (Continued) 

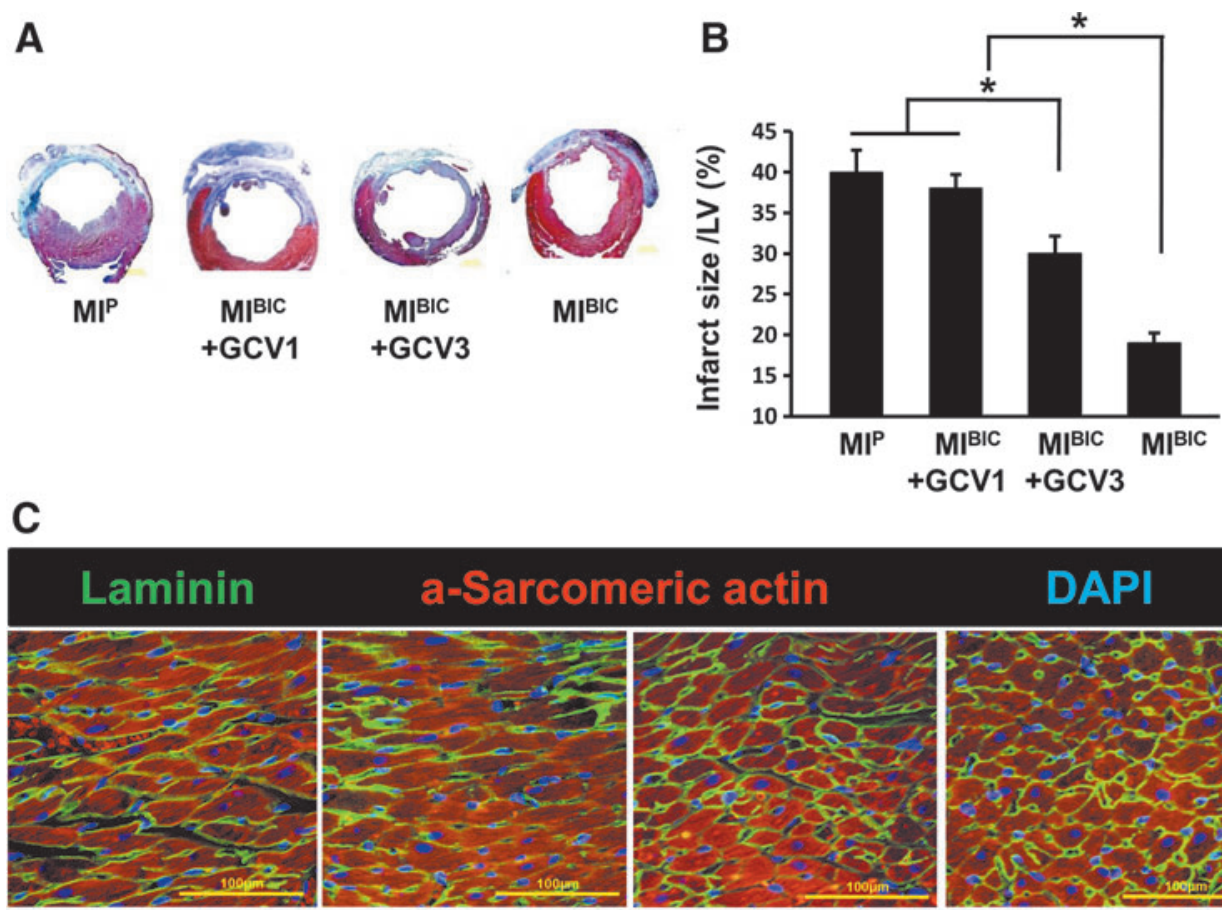

MIP

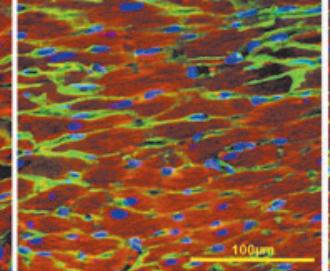

$M I^{\mathrm{BIC}}+\mathrm{GCV} 1$

$\mathrm{MI}^{\mathrm{BIC}}+\mathrm{GCV} 3$

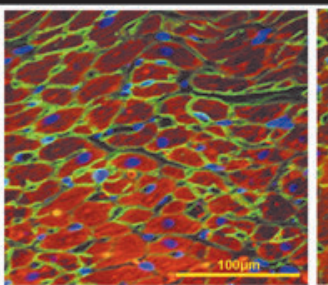

DAPI
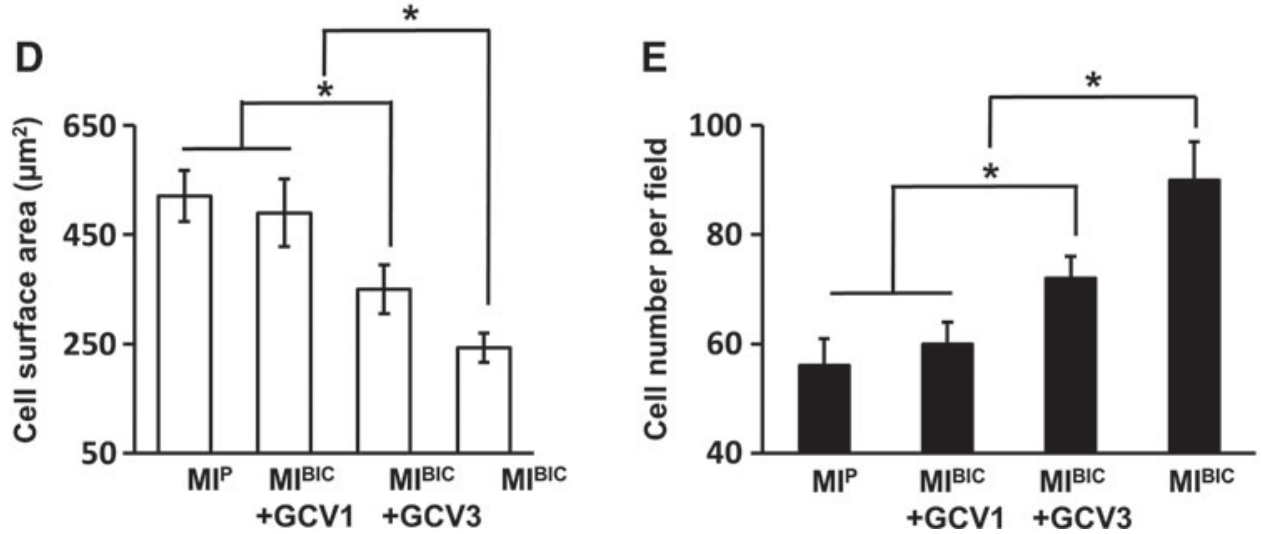

FIG. 6. Effect of selective, timed elimination of cardiovascular cells in LV fibrosis. (A) Masson-Trichome staining on $5-\mu \mathrm{m}$ sections from heart slices in various treatment groups. MI, myocardial infarction. (B) Infarct size (\% of LV) in hearts at 5 weeks after permanent LAD occlusion in various treatment groups. All values were expressed as mean \pm SEM. $n=6$ for each group. ${ }^{*} p \leq 0.05$ was considered statistically significant. (C) Representative images of peri-infarction border zone sections stained for $\alpha$-Sarcomeric actin (red), nuclei (DAPI, blue), and laminin (green). (D, E) Quantitative data for cardiomyocyte size using cell surface area and cell density (nuclei per high field). All values were expressed as mean \pm SEM. ${ }^{*} p<0.05$ was considered statistically significant; $n=6$ in each group. (F, G) Quantitative analysis for anterior wall thickness and posterior wall thickness. ${ }^{*} p \leq 0.05$ was considered statistically significant. $n=6$ for each group. (H-K) Quantitative analysis (LVDd, LVDs, LVEF, and LVFS) of heart function at 5 weeks after permanent LAD occlusion in various treatment groups. Left ventricular end-diastolic dimension (LVDd), left ventricular end-systolic dimension (LVDs), $\mathrm{EF}$, ejection fraction index; FS, fractional shortening. All values expressed as mean $\pm \mathrm{SEM}$. ${ }^{*} p \leq 0.05$ was considered statistically significant. $n=6$ for each group. (L) TUNEL positive nuclei (white arrows) in the peri-infarct area at 7 days after cell patch implantation and quantitative data in various treatment groups. All values were expressed as mean \pm SEM. $n=6$ for each group. ${ }^{*} p \leq 0.05$ was considered statistically significant. MI ${ }^{\mathrm{P}}$ group: MI-operated rats with peritoneum patch without cells; MI ${ }^{\mathrm{BC}}$ group: MI-operated rats with bi-cell $(\mathrm{CM}+\mathrm{EC})$-seeded peritoneum patch; $\mathrm{MI}^{\mathrm{BIC}}+\mathrm{GCV} 1$ group: MIoperated rats with bi-cell patch given GCV in first week; $\mathrm{MI}^{\mathrm{BIC}}+\mathrm{GCV} 1$ group: MI-operated rats with bi-cell patch given GCV in third week. 

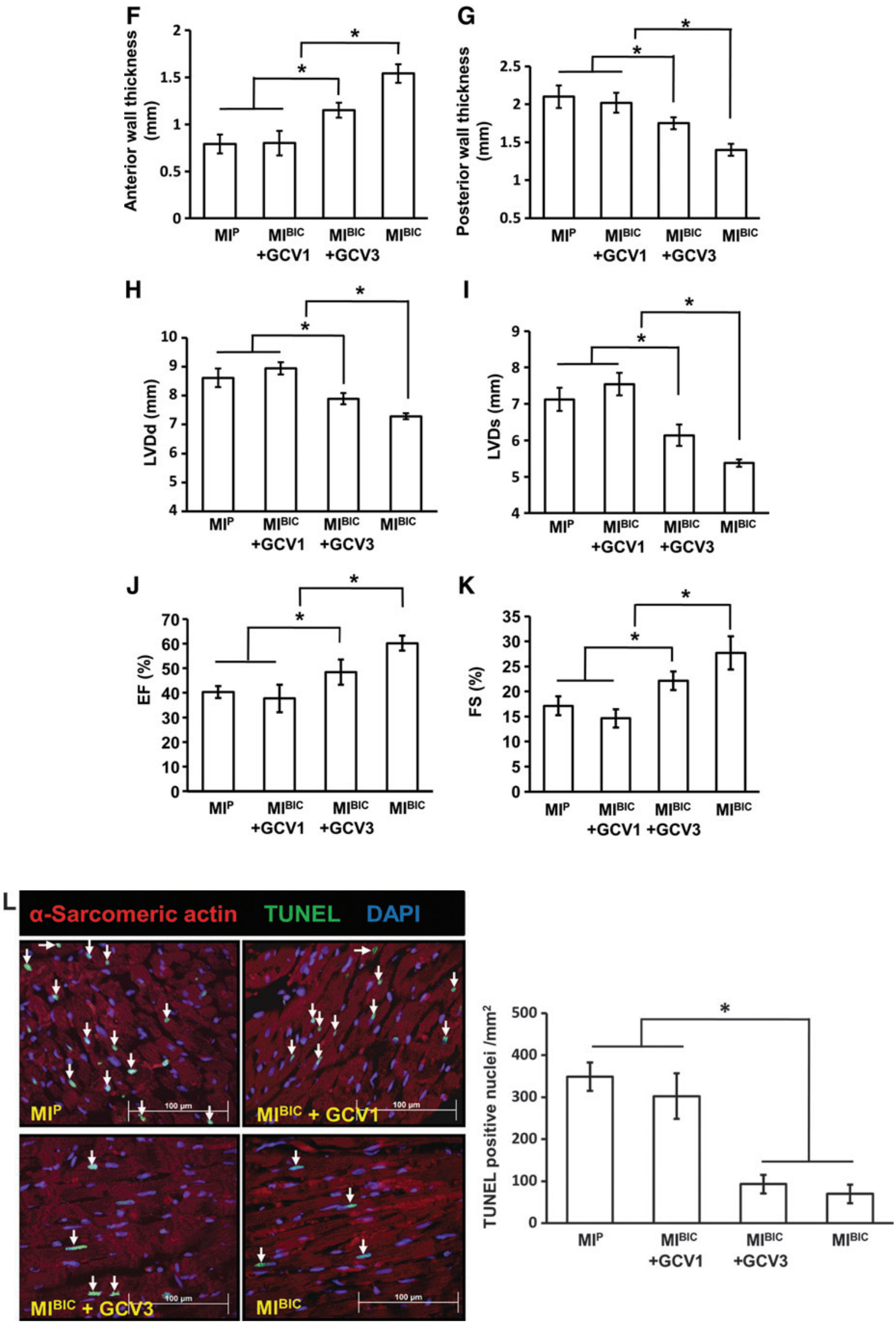

FIG. 6. (Continued) 
available online at www.liebertpub.com/ars). In addition, when the BIC group underwent GCV treatment, the LV anterior wall thickness was significantly reduced in the MI${ }^{\mathrm{BIC}}+\mathrm{GCV} 3$ group, especially in the $\mathrm{MI}^{\mathrm{BIC}}+\mathrm{GCV} 1$ group, which is similar to the MIP group. In the absence of GCV, the LV anterior wall thinning was significantly preserved in the BIC treated group, as compared with other groups (Fig. 6F). Importantly, reduced compensatory hypertrophy was evidenced in the lower LV posterior wall thickness in diastole in BIC-treated hearts (Fig. 6G), which was reversed by GCV administration.

Large numbers of cells undergoing apoptosis, as reflected by TUNEL-positive nuclei, were observed in the periinfarcted area at 7 days after cell patch implantation in the $\mathrm{MI}^{\mathrm{P}}$ group (Fig. 6L, arrow). Similar numbers of cells were observed in the $\mathrm{MI}^{\mathrm{BIC}}+\mathrm{GCV} 1$ group using TUNEL-positive nuclei. However, the number of cells with TUNEL-positive nuclei was significantly reduced in the $\mathrm{MI}^{\mathrm{BIC}}+\mathrm{GCV} 3$ group. Numbers of nuclei reached counts similar to those seen in the $\mathrm{MI}^{\mathrm{BIC}}$ group.

\section{Discussion}

TK transgenic iPSC were generated, and cell viability was controlled by generation of intracellular toxic molecules after GCV administration. These iPSC-derived cardiovascular cells were then prepared into a cell patch for direct application onto the epicardial surface of an infarcted rat heart. Experimental results obtained in this study provide evidence that (i) iPSC-derived cardiovascular cells - both CM and ECs - were the predominant contributors to cardiac functional improvement after acute MI; (ii) the depletion of cardiovascular cells by GCV administration at the first week was followed by a deterioration in LV contractile function, associated with a reduction of cell-associated paracrine factors and with a decrease in vascular density; (iii) the depletion of iPSC-derived cardiovascular cells by GCV administrated at the third week reduced LV contractile function, as well as the vascular density; and (iv) the extent of LV contractile function reduction after GCV treatment at the third week was less than that seen with treatment at the first week. These findings support a 2-phase overlapping mechanism by which the iPSC-derived CM and EC delivered in the cell patch over the infarcted myocardium promote a reverse myocardial remodeling in which the therapeutic effect of iPSC-derived cardiovascular cells in the rat MI model is in the early phase due to paracrine factors released from iPSC, with a sustained improvement during the second phase when cardiovascular cells remain viable and continue to express these factors.

Previous studies with intravascular and intramyocardial injections of stem cells demonstrated functional restoration of the LV after MI (10, 11, 13). However, it is difficult to control the delivery of grafted cell populations to the target tissue using such methods. Optimization of the cell delivery methods is essential to enhance functional benefit in such cellular therapies. The peritoneum patch containing iPSC provides a supportive micro-environment for cell engraftment, migration, and survival within the infarcted myocardium (4). Nevertheless, the precise cellular mechanism underlying the salutary effects associated with such cell therapy still remains unclear. The suicide gene system under the control of cardiomyocyte (NCX1) and endothelium ( VEcadherin) specific promoters provided a useful tool for elucidation of these mechanisms, by enabling selective and timed ablation of cell lines derived from iPSC.

The paracrine mechanism for the therapeutic effects of transplanted stem/PCs has been proposed in cardiovascular diseases (3). We, therefore, studied paracrine factor expression in the $\mathrm{CM}$ and $\mathrm{EC}$ under normoxia and anoxia in vitro (Fig. 3A, B). Among myriad putative paracrine factors, $I G F$ $1 \propto$ possesses broad biological effects, including those such as angiogenesis and cardiac stem cell survival (14). IGF-1 $\alpha$ overexpression or local delivery of $I G F-1 \alpha$ improves myocyte survival and protects against ventricular dilatation after injection (5). VEGF is also documented to induce angiogenesis (1). In addition, $T G F-\beta 1$ is markedly activated in the infarcted myocardium, which is crucial for repression of inflammatory gene synthesis and cardiomyocyte hypertrophy (2). Taken together, this information suggested that paracrine factors released by iPSC-derived cardiovascular cells under anoxia conditions might initiate a cytokine signaling cascade which promotes cardiac repair and regeneration after BIC transplantation. To further investigate the paracrine effect in vivo after BIC transplantation in the setting of MI, we employed a strategy of "suicide gene" activation by GCV administration.

At various time points after BIC transplantation onto the MI heart, the mRNA levels of these paracrine factors were found to be significantly increased compared with those in the $\mathrm{MI}^{\mathrm{P}}$ group, with the peak values appearing before or on the seventh day, and returning to the baseline by 21 days. The transduction of TK gene under the control of a cardiacspecific promoter (NXC1 promoter) or endothelia-specific promoter (VE-cadherin promoter) was expressed in iPSCderived CM and EC. When GCV was acquired by CM or EC, the TK gene was activated and cell death ensued. Our data confirmed the effectiveness of this suicide gene approach when GCV administration specifically resulted in the death of $\mathrm{CM}$ expressing TK and EC expressing TK. In vivo, when GCV was administrated during the first week, severely cardiomyocyte apoptosis ensued. Peak levels of growth factors were significantly reduced, and the mRNA levels in the GCV treatment group were reduced to those in MI control group, leading to severe cardiomyocyte apoptosis. In addition, the significantly increased vascular density associated with the application of BIC patch was completely abolished by GCV administration during the first week. Thus, paracrine factors released by iPSC-derived cardiovascular cells contribute to a reverse myocardial remodeling process during a critical period of infarcted heart repair. During the later phase (at week 3 ), we noted that vascular density was not abolished by GCV administration, but rather was partially preserved and less cardiomyocyte apoptosis was evident in the BIC treated hearts. As other studies have demonstrated, paracrine factors released from transplanted stem/PCs can also augment various humoral factors of recipient heart tissue, thereby providing an additional favorable environment for angiogenesis and heart function improvement (3). These additional signaling molecules might participate as supplementary mechanisms to explain the difference we observed when GCV was administrated at different time points. In addition to the paracrine mechanism we advocated based on our data in these studies, a comparative analysis of data on iPSC 
engraftment and cardiovascular cell differentiation provided a second and potentially equally important therapeutic mechanism for ischemic myocardium repair and regeneration. The angiomyogenesis observed was abrogated similarly using the suicide gene approach under the control of a cardiac-specific promoter ( $N X C 1$ promoter) or endotheliaspecific promoter (VE-cadherin promoter). The GFP and $\alpha$-sarcomeric-actin-positive cells $\left(\mathrm{GFP}^{+} / \mathrm{actin}^{+}\right)$were used to trace the penetration of iPSC-derived CM from the cell patch into the infarcted area. We confirmed that grafted cells from the cell patch had entered into the infarcted region after patch implantation, while grafted cells in the patch were significantly destroyed after GCV had been administered during either the first or third weeks (Figs. 4 and 5).

To assess the specific therapeutic mechanism(s) of BIC, cardiac function was analyzed using echocardiography combined with the suicide gene approach. Differential regional patterns after anterior infarction were induced by left anterior descending artery (LAD) ligation for systolic function, muscle hypertrophy, which accompanied serial changes in ventricular chamber volumes (15). As revealed by echocardiography analysis, LV remodeling was significantly improved in the $\mathrm{MI}^{\mathrm{BIC}}$ group as indicated by a reduction of $\mathrm{LV}$ chamber volume, an increase in LV EF, FS, and anterior wall thickness as compared with other groups. Although TK activated by GCV severely reversed the benefits of BIC (as evidenced by worsening cardiac function and LV wall thinning), the effect of TK activation at two time points is different. Further insights were obtained by measuring these cardiovascular cell differentiation levels in various groups. In the absence of GCV, BIC treatment enhanced the migration and cardiovascular cell differentiation of iPSC in the infarcted heart. This finding was consistent with the echocardiography results. Hypertrophy of surviving CM is a major compensatory mechanism post-MI, but in the longer term, it can be associated with an increased risk of heart failure and malignant arrhythmia and metabolic activity (17). The reduced compensatory hypertrophy and decreased LV posterior wall thickness in the BIC-treated group highlights the anti-remodeling effect of BIC treatment that resulted from enhanced angiomyogenesis in combination with a favorable paracrine effect rather than from cardiomyocyte hypertrophy. In contrast, GCV administration in the third week only partially reversed BIC-induced beneficial effects when the suicide gene was activated to specifically kill the differentiated cardiovascular cells. This result supported an important role of iPSC-cardiovascular cell differentiation in MI therapy and reversal of heart tissue remodeling after MI. It has been reported that overexpression of angiogenesis factors in the myocardium contributed to functional restoration post-MI (16). In this study, although heart function was improved in the $\mathrm{MI}^{\mathrm{CM}}$ or $\mathrm{MI}^{\mathrm{EC}}$ group as compared with the $\mathrm{MI}^{\mathrm{P}}$ group, no significant difference was detected after 4 weeks between these two groups, which was similar to the $\mathrm{MI}^{\mathrm{BIC}}+\mathrm{GCV} 3$ group.

In conclusion, implantation of a cell patch containing cardiovascular cells (CM and EC) derived from iPSC can significantly improve contractile function of an infarcted heart. The specific gene suicide approach with sequencing ablation targeted by GCV enabled us to determine that the early salutary effects of cardiovascular cells derived from
iPSC on angiogenesis and consequent improvements in LV function were largely due to paracrine effects; while the long-term, potentially important, therapeutic effects were largely due to the new EC and CM populations derived from iPSC.

\section{Materials and Methods}

\section{Laboratory animals}

All research protocols conformed to the Guidelines for the Care and Use of Laboratory Animals published by the National Institutes of Health (National Academies Press, eighth edition, 2011). All animal use protocols and methods of euthanasia (pentobarbital overdose followed by thoracotomy) used in this study were preapproved by the University of Cincinnati Animal Care and Use Committee. An independent review and approval of cell and virus methods used in this study was conducted by the Institutional Biosafety Committee (IBC).

\section{Experiment design}

In vitro: iPSC-derived cardiovascular cells were induced by genetic modification with herpes simplex virus TK "suicide" gene driven by cardiac NCX1 (CM) or endothelial VE-cadherin promoter (EC). The effectiveness of the suicide gene approach and iPSC differentiation potential were examined by RT-PCR, immunofluorescence staining, and FACS. To induce growth factor (IGF-1 $\alpha$, $V E G F$, and $T G F-\beta 1$ ) release, $\mathrm{CM}$ and $\mathrm{EC}$ were exposed to the anoxia condition, followed by qRT-PCR analysis. In vivo: An ischemia-induced MI model was developed in 10-12 week-old nude rats (Charles River), and the animals were randomly divided into the following groups: (i) $\mathrm{MI}^{\mathrm{P}}$ group (MI-operated rats with peritoneum patch without cells); (ii) $\mathrm{MI}^{\mathrm{BIC}}$ group (MI-operated rats with bi-cell $(\mathrm{CM}+\mathrm{EC})$-seeded peritoneum patch); (iii) $\mathrm{MI}^{\mathrm{BIC}}+\mathrm{GCV} 1$ group (MI-operated rats with bi-cell patch given GCV in first week); (iv) $\mathrm{MI}^{\mathrm{BIC}}+\mathrm{GCV} 1$ group (MI-operated rats with bi-cell patch given GCV in third week); (v) $\mathrm{MI}^{\mathrm{EC}}$ group (MI-operated rats with EC patch); and (vi) $\mathrm{MI}^{\mathrm{CM}}$ group (MI-operated rats with $\mathrm{CM}$ patch). Echocardiography was performed before or at week 4 after cell patch transplantation. After 4 weeks for patch treatment, hearts were fixed for immunohistochemical assessment of angiomyogenesis, hypertrophy, as well as Masson's Trichrome staining for infarct size assay. Additional hearts in various groups at day $0,3,7,14$, and 28 were harvested and followed by Western blotting and qPCR for in vivo growth factor release profile analysis. To evaluate cardiomyocyte apoptosis at 1 week after transplantation, the heat tissue was fixed for TUNEL analysis.

\section{Lentiviral vectors and transduction}

The multiple cloning site of pCDH-GFP between EcoRI and NotI was replaced with TK gene obtained from pORFTK (Invivogen) to construct the vector pCDH-TK-GFP. Sodium/Calcium exchanger (NCX1) promoter was then subcloned into pCDH-TK-GFP between CalI and EcoRI restriction enzyme sites replacing the CMV promoter ( $\mathrm{pCDH}-$ NCX1-TK-GFP), while a short fragment was subcloned into the same sites to produce a promoterless vector as the 
negative control (pCDH-Null-TK-GFP). The endothelialspecific VE-cadherin promoter was subcloned into the sites to produce pCDH-VE-TK-GFP as described (12). The identity of the promoter was confirmed by sequencing (Genewiz). Pseudoviral particles were produced in 293 YN cells using pPACK lentivector Packaging Kit according to the manufacturer's instructions. Target cells were transduced with pseudoviral stock, and the transgenic positive cellular clone was selected by puromycin ( $5 \mu \mathrm{g} / \mathrm{ml}$; Sigma-Aldrich).

\section{Cell culture and suicide gene approach}

Neo-CM were isolated from ventricles of 2-day-old neonatal Sprague-Dawley rats using a neonatal cardiomyocyte isolation kit (Worthington Biochemical) as described (4). A rat EC line, YPEN-1, was maintained with Dulbecco's modified Eagle's medium (DMEM) supplemented with sodium bicarbonate $(1.5 \mathrm{mg} / \mathrm{ml}), 0.1 \mathrm{~m} M$ nonessential amino acids, $1.0 \mathrm{~m} M$ sodium pyruvate, heparin $(30 \mathrm{~g} / \mathrm{ml})$, and $5 \%$ fetal bovine serum (FBS). CM and YPEN-1 were incubated in viral supernatants containing pCDH-Null-TK-GFP, pCDH-CMVTK-GFP, pCDH-NCX1-TK-GFP, or pCDH-VE-TK-GFP, respectively. Cells were then treated with $100 \mu M \mathrm{GCV}$ for 4 days. Viable cells were identified by GFP or immunostaining and counted under a microscope at 200-magnification.

\section{Anoxia and real-time PCR}

The iPSC-derived CM and EC were seeded and cultured for $24 \mathrm{~h}$ before anoxia. The medium containing serum was replaced with glucose-free and serum-free DMEM and then subjected to anoxia $\left(5 \% \mathrm{CO}_{2} / 95 \% \mathrm{~N}_{2}\right)$ for $4 \mathrm{~h}$ in an anoxia chamber (Forma Scientific Corp.). The cells were collected for total RNA extraction later using TRIzol Reagent (Ambion, Life Technologies). Total RNA was used for reverse transcription in a miScript II RT kit (Qiagen). The primer sequence for the genes used in qPCR is listed below. qPCR was performed using miScript SYBR Green PCR kit (Qiagen). The expression of genes of interest was normalized to that of $\beta$-actin. The primers for $\mathrm{qPCR}$ were synthesized as follows: $I G F-1 \alpha$ forward primer: $5^{\prime}$ - TGAGCTGGTG GATGCTCTTCAGTT-3', reverse primer: 5'- TCATCCAC AATGCCTGTCTGAGGT-3'; VEGF forward primer: 5'GCA ACACCAAGTCCGAATGCAGAT-3', reverse primer: 5'-TCTGGCTTCACAGCACTC TCCTTT-3'; TGF- $\beta 1$ forward primer: 5'- ACCAACTACTGCTTCAGCTCCACA -3', reverse primer: 5'- TGTACTGTGTGTCCAGGCTCCA AA-3'; Oct4 forward primer: 5'- TGGAGGAAGCCGACAA CAATGAGA-3', reverse primer: 5'-TGGCGATGTGAGTG ATCTGCTGTA-3'; Sox2 forward primer: 5' -CAGGAGTT GTCAAGGCAGAGA-3', reverse primer: 5'-CTTAAGCCT CGGGCTCCAAA-3'; Klf4 forward primer: 5'- CCCAAACC TTGCCGCATTAAGGAA-3', reverse primer: $5^{\prime}$ - ACACCC TTGGATGCTCTTCTTGGA-3'; GATA4 forward primer: 5'TCTGGCTGGCCGAGAGCAGT-3', reverse primer: 5'-GGC TGTGCAGGA CTGGGCTG-3'; GATA2 forward primer: 5'GGAGAAAGGAGTAGGCAAGAAG-3', reverse primer: 5'CCCAAGAACACAAATAGCACAC-3'; $N k x 2.5$ forward primer: 5'- TGGGTCTCAATGCCTATGGCTACA-3', reverse primer: 5'-GACGCCAAAGTTCAC GAAGTTGCT-3'; $\beta$-Actin forward primer: $5^{\prime}$ - TGTCATCCTCCCAATCCCTC AGAA-3', reverse primer: 5'- TGTGGTGCCAGATCTTCT CCATGT-3'.

\section{iPSC culture and cell differentiation}

Mouse iPSC were maintained as described (4). Briefly, iPSC were maintained in DMEM containing $15 \%$ knockout serum replacement, $0.1 \mathrm{~m} M$ nonessential amino acids, $2 \mathrm{~m} M$ L-glutamine, $0.1 \% \mathrm{~m} M \beta$-mercaptoethanol, and $1000 \mathrm{U} / \mathrm{ml}$ leukemia inhibitory factor (LIF) on feeder layer of mitomycin C-treated mouse embryonic fibroblast cells. Cell differentiation was induced using the hanging drop method (8). iPSC were cultured in hanging drops of 500 cells per $20 \mu \mathrm{l}$ of differentiation medium (composition identical to maintenance medium but without LIF and with $10 \%$ FBS replacing knockout serum) to form embryonic bodies (EBs). On day 3, EBs were transferred into fresh differentiation medium for suspension cultivation in nonadhering petri dishes. After 5 days, EBs were transferred to $0.1 \%$ gelatin-coated dishes. Medium was changed the next day and then changed every other day to maintain viable cells.

\section{Reverse transcription-polymerase chain reaction}

For RT-PCR analysis, RNA was isolated from undifferentiated iPSC (Day 0) and differentiated EBs with Trizol Reagent. RT into complementary DNA was conducted with miScript II RT kit (Qiagen). The PCR-related primers were the same as those used previously for anoxia and real-time PCR. Each RT-PCR was performed under the following conditions: $30 \mathrm{~s}$ at $94^{\circ} \mathrm{C}, 30 \mathrm{~s}$ at $55^{\circ} \mathrm{C}$, and $1 \mathrm{~min}$ at $72^{\circ} \mathrm{C}$. The details were described in our previous publication (4).

\section{Western blot analysis}

Western blotting was performed in heart tissues of various treatment groups to determine the expression of $I G F-1 \alpha$, $V E G F$, and $T G F-\beta 1$ at different time points (9). Briefly, after electrophoresis, transformation, and immunoblotting, the samples on PVDF membranes were visualized with the ECL plus kit (Bio-Rad). Antibodies against $I G F-1 \alpha, V E G F$ were from Santa Cruz Biotechnology. Antibody against TGF- $\beta 1$ was from Cell Signaling Technology.

\section{Immunocytological staining}

Several beating EBs (day 15 of differentiation) were selected for dissociation using $10 \mathrm{mg} / \mathrm{ml}$ collagenase IV for 3 to $4 \mathrm{~h}$ at $37^{\circ} \mathrm{C}$. Dissociated cells were seeded into $0.1 \%$ gelatincoated dishes. After 2 or 3 days, the cells were fixed in $4 \%$ paraformaldehyde for $30 \mathrm{~min}$ and subsequently permeabilized with $0.2 \%$ Triton X-100 at room temperature. The fixed/ permeabilized cells were then incubated at $4{ }^{\circ} \mathrm{C}$ overnight with monoclonal anti-cardiac actin. Tritc-conjugated donkey anti-mouse IgG was applied to the cells for $1 \mathrm{~h}$ at room temperature. Nuclei were stained with DAPI for $5 \mathrm{~min}$ at room temperature.

\section{Quantification of cardiovascular cell differentiation by flow cytometry}

Differentiated iPSC were trypsinized and re-suspended as single cells in a glass tube at 15 days after differentiation had begun. Cells were fixed on ice with $4 \%$ paraformaldehyde for $30 \mathrm{~min}$ and permeabilized in $0.1 \%$ saponin for $30 \mathrm{~min}$. After incubation with $1 \% \mathrm{FBS}$ on ice for $30 \mathrm{~min}$, mouse anti-a-sarcomeric-actin primary antibody was added. 
After incubating overnight at $4^{\circ} \mathrm{C}$, cells were washed and incubated with donkey anti-mouse IgG-Cy5 for $1 \mathrm{~h}$ at room temperature. Cells were incubated with anti CD31 overnight at $4^{\circ} \mathrm{C}$ to enable ECs counting. After thorough washing with $\mathrm{PBS}$, cells were re-suspended in $0.3 \mathrm{ml}$ PBS. Samples were analyzed using an FACS Aria instrument (BD Biosciences). Cells with an adequate size and granularity were accounted for in the statistical analysis. The percentage of $\alpha$-actin cells/ total cells was calculated.

\section{MI model and cell patch implantation}

An MI model was developed in nude rats (female), as previously described (6). Briefly, nude rats (200-250 g) were anesthetized by spontaneous inhalation and maintained under general anesthesia with 1-2\% isoflurane. Animals were mechanically ventilated using a rodent ventilator (Model 683; Harvard Apparatus) connected to an endotracheal tube. The heart was exposed by a left side limited thoracotomy, and the LAD was ligated with a $6-0$ polyester suture $1 \mathrm{~mm}$ from the apex of the normally positioned left auricle.

iPSC were transduced with pseudoviral particles containing pCDH-NCX1-TK-GFP and pCDH-VE-TK-GFP at 2 days after $\mathrm{EBs}$ that were attached were $0.1 \%$ gelatin coated. $\mathrm{CM}$ and EC $\left(2 \times 10^{6}\right)$ with a 1:1 ratio carrying specific suicide genes were seeded on a peritoneal cell patch ${ }^{2}$. One week after MI, the left thoracic cavity was reopened. Cell patches were applied to the surface of LAD distribution overarching the MI area by surface tension of the isolated peritoneum without any suture. The rats were simultaneously injected intraperitoneally with $10 \mathrm{mg} / \mathrm{kg} \mathrm{GCV}$ daily for 7 days, and separate groups were injected at the third week.

\section{Immunohistochemistry of rat hearts}

After coronary perfusion with fixative solution, the heart was removed and fixed by immersion in $4 \%$ PFA in PBS. To measure infarct size at 4 weeks after MI, the LV cross-sections from mid-LV to apex stained with Masson's Trichrome were used to quantify infarct size in the left ventricle in various treatment groups. LV infarct area and total LV area of each image were measured using the Image-Pro-Plus (Media Cybernetics, Inc.), and the infarct area was reported as a percentage of the total LV area. To determine cardiomyocyte hypertrophy post-MI, hearts after 4 weeks of different treatments were stained with $\alpha$-sarcomeric actin (for cardiomyocyte) and laminin (for the surface cell membrane). Myocyte area was measured in captured images using Image J (17). LV sections from border zone of infarcted hearts were used to quantify capillary density after staining with $v W F$ antibody (Dako) as previously described (9). To quantify apoptotic CM, additional rat hearts were removed at 1 week after cell patch implantation, fixed with $0.5 \%$ PFA in 5\% sucrose, routinely frozen embedded in OCT, and processed for sectioning and staining with TUNEL and $\alpha$-sarcomeric actin (Sigma-Aldrich) as previously described (6). TUNEL was performed using an In Situ Cell Death Detection kit, Fluorescein (Roche Applied Science) as per the manufacturer's protocol. DAPI was used for nuclear counterstaining. For angiomyogenesis analysis, additional hearts were subjected to cryosection. The number of $\mathrm{GFP}^{+}$cells per $\mathrm{mm}^{2}$ in the infarcted area was counted to evaluate cell migration from the cell patch. Four fields of each section were examined for quantification. Fluorescent imaging was performed with an Olympus BX41 microscope (Olympus America, Inc.) equipped with an epifluorescence attachment. Images were recorded using a digital camera and analyzed using MagnaFire ${ }^{\mathrm{TM}} 2.1$ software.

\section{Echocardiography}

Transthoracic echocardiography (iE33 Ultrasound System; Phillips) was performed with a $15-\mathrm{MHz}$ probe. Hearts were imaged in 2D long-axis view at the level of the greatest LV diameter in animals under light general anesthesia (13). This view was used to position the M-mode cursor perpendicular to the LV anterior and posterior walls. LV enddiastolic and end-systolic diameters were measured from M-mode recordings. LV ejection fraction (EF) was calculated as $\mathrm{EF}($ as a\%) = [left ventricular end-diastolic dimension $(\mathrm{LVDd})^{3}$ minus left ventricular end systolic dimension $(\mathrm{LVDs})^{3} /(\mathrm{LVDd})^{3} \times 100$. LV fractional shortening (FS as a\%) was determined as [(LVDd-LVDs)/LVDd $\times 100$. All measurements were performed according to the American Society for Echocardiography leading-edge technique standards, and averaged over three consecutive cardiac cycles.

\section{Statistical analysis}

Results were statistically analyzed with the use of the StatView 5.0 software package (Abacus Concepts, Inc.). All values are expressed as mean \pm SEM. Student's $t$-test was applied to compare growth factor expression between normoxia and anoxia condition. Two-way ANOVA were used to analyze the growth factor expression in various treatment groups at different time points. One-way ANOVA was applied to analyze angiomyogenesis, infarct size, echocardiographic data, and TUNEL assay data. After ANOVA, the post hoc Bonferroni was performed for comparisons of multiple groups. A value of $p<0.05$ was considered statistically significant.

\section{Acknowledgments}

The authors wish to recognize Christian Paul for technical assistance. This work was funded by National Institutes of Health grants HL089824, HL-110740, and HL107957 (Y. Wang).

\section{Author Disclosure Statement}

The authors declare that they have no competing interests to disclose.

\section{References}

1. Albrecht-Schgoer K, Schgoer W, Holfeld J, Theurl M, Wiedemann D, Steger C, Gupta R, Semsroth S, FischerColbrie R, Beer AG, Stanzl U, Huber E, Misener S, Dejaco $\mathrm{D}$, Kishore R, Pachinger O, Grimm M, Bonaros N, and Kirchmair R. The angiogenic factor secretoneurin induces coronary angiogenesis in a model of myocardial infarction by stimulation of vascular endothelial growth factor signaling in endothelial cells. Circulation 126: 2491-2501, 2012.

2. Bujak M and Frangogiannis NG. The role of TGF-beta signaling in myocardial infarction and cardiac remodeling. Cardiovasc Res 74: 184-195, 2007. 
3. Cho HJ, Lee N, Lee JY, Choi YJ, Ii M, Wecker A, Jeong JO, Curry C, Qin G, and Yoon YS. Role of host tissues for sustained humoral effects after endothelial progenitor cell transplantation into the ischemic heart. J Exp Med 204: 3257-3269, 2007.

4. Dai B, Huang W, Xu M, Millard RW, Gao MH, Hammond HK, Menick DR, Ashraf M, and Wang Y. Reduced collagen deposition in infarcted myocardium facilitates induced pluripotent stem cell engraftment and angiomyogenesis for improvement of left ventricular function. J Am Coll Cardiol 58: 2118-2127, 2011.

5. Davis ME, Hsieh PC, Takahashi T, Song Q, Zhang S, Kamm RD, Grodzinsky AJ, Anversa P, and Lee RT. Local myocardial insulin-like growth factor 1 (IGF-1) delivery with biotinylated peptide nanofibers improves cell therapy for myocardial infarction. Proc Natl Acad Sci U S A 103: 8155-8160, 2006.

6. Huang W, Dai B, Wen Z, Millard RW, Yu XY, Luther K, Xu M, Zhao TC, Yang HT, Qi Z, Lasance K, Ashraf M, and Wang Y. Molecular strategy to reduce in vivo collagen barrier promotes entry of NCX1 positive inducible pluripotent stem cells (iPSC(NCX $(1)(+))$ ) into ischemic (or injured) myocardium. PLoS One 8: e70023, 2013.

7. Krankel N, Spinetti G, Amadesi S, and Madeddu P. Targeting stem cell niches and trafficking for cardiovascular therapy. Pharmacol Ther 129: 62-81, 2011.

8. Kurosawa H. Methods for inducing embryoid body formation: in vitro differentiation system of embryonic stem cells. J Biosci Bioeng 103: 389-398, 2007.

9. Liang J, Huang W, Yu X, Ashraf A, Wary KK, Xu M, Millard RW, Ashraf M, and Wang Y. Suicide gene reveals the myocardial neovascularization role of mesenchymal stem cells overexpressing CXCR4 (MSC(CXCR4)). PLoS One 7: e46158, 2012.

10. Mirotsou M, Jayawardena TM, Schmeckpeper J, Gnecchi $\mathrm{M}$, and Dzau VJ. Paracrine mechanisms of stem cell reparative and regenerative actions in the heart. $J$ Mol Cell Cardiol 50: 280-289, 2011.

11. Miyagawa S, Sawa Y, Fukuda K, Hisaka Y, Taketani S, Memon IA, and Matsuda H. Angiogenic gene cell therapy using suicide gene system regulates the effect of angiogenesis in infarcted rat heart. Transplantation 81: 902-907, 2006.

12. Nelson TJ, Martinez-Fernandez A, Yamada S, Perez-Terzic C, Ikeda Y, and Terzic A. Repair of acute myocardial infarction by human stemness factors induced pluripotent stem cells. Circulation 120: 408-416, 2009.

13. Robinton DA and Daley GQ. The promise of induced pluripotent stem cells in research and therapy. Nature 481: 295-305, 2012.

14. Santini MP, Tsao L, Monassier L, Theodoropoulos C, Carter J, Lara-Pezzi E, Slonimsky E, Salimova E, Delafontaine P, Song YH, Bergmann M, Freund C, Suzuki K, and Rosenthal N. Enhancing repair of the mammalian heart. Circ Res 100: 1732-1740, 2007.

15. Sehgal M, Hirose K, Reed JE, and Rumberger JA. Regional left ventricular wall thickness and systolic function during the first year after index anterior wall myocardial infarction: serial effects of ventricular remodeling. Int J Cardiol 53: 45-54, 1996.

16. Tirziu D, Chorianopoulos E, Moodie KL, Palac RT, Zhuang ZW, Tjwa M, Roncal C, Eriksson U, Fu Q, El- fenbein A, Hall AE, Carmeliet P, Moons L, and Simons M. Myocardial hypertrophy in the absence of external stimuli is induced by angiogenesis in mice. J Clin Invest 117: 3188-3197, 2007.

17. Tseliou E, de Couto G, Terrovitis J, Sun B, Weixin L, Marban L, and Marban E. Angiogenesis, cardiomyocyte proliferation and anti-fibrotic effects underlie structural preservation post-infarction by intramyocardially-injected cardiospheres. PLoS One 9: e88590, 2014.

18. Zhao T, Zhang D, Millard RW, Ashraf M, and Wang Y. Stem cell homing and angiomyogenesis in transplanted hearts are enhanced by combined intramyocardial SDF1alpha delivery and endogenous cytokine signaling. Am $J$ Physiol Heart Circ Physiol 296: H976-H986, 2009.

Address correspondence to: Dr. Yigang Wang

Department of Pathology and Laboratory Medicine University of Cincinnati Medical Center 231 Albert Sabin Way Cincinnati, OH 45267-0529

E-mail: yi-gang.wang@uc.edu

Date of first submission to ARS Central, November 12, 2013; date of final revised submission, April 7, 2014; date of acceptance, April 29, 2014.

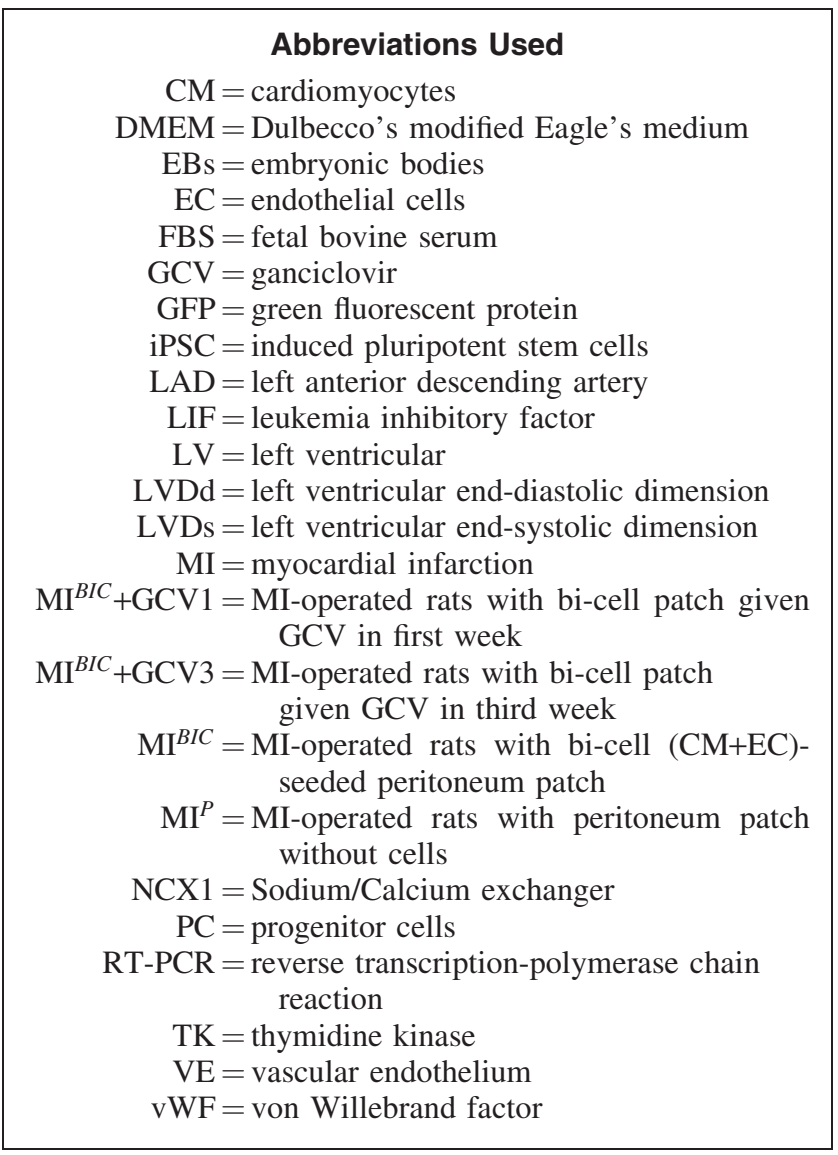

